\title{
Time- and energy-resolved measurements of ion beams emitted from Plasma-Focus type discharges
}

\author{
R. Kwiatkowski ${ }^{\mathrm{a} 1}$, K. Czaus $^{\mathrm{a}}$, M. Paduch ${ }^{\mathrm{b}}$, M. J. Sadowski ${ }^{\mathrm{ab}}$, \\ E. Skladnik-Sadowska ${ }^{a}$ and E. Zielinska ${ }^{b}$ \\ ${ }^{a}$ National Centre for Nuclear Research (NCBJ), \\ 05-400 Otwock, Poland \\ ${ }^{b}$ Institute of Plasma Physics and Laser Microfusion (IFPiLM), \\ 01-497 Warsaw, Poland \\ E-mail: Roch.Kwiatkowski@ncbj.gov.pl
}

The paper presents results of research on temporal- and energetic-characteristics of fast ion beams emitted from high-current discharges of the Plasma-Focus (PF) type. The measurements were performed within a modified DPF-1000U facility, operated at IFPiLM in Warsaw, Poland. The device was equipped with a fast-acting gas valve which was located on the axis of the inner electrode and could inject a small volume of a chosen gas in front of this electrode. The discharges were initiated at various initial deuterium pressures, without or with the use of the gas-puffing valve. Such discharges emitted intense beams of accelerated primary ions and X-ray pulses as well as fusion products.

Measurements of the fast ion beams were performed by means of a Thomson-parabola
spectrometer equipped with nuclear track detectors or with miniature scintillation detectors.
Energy spectra of the investigated ion beams were recorded using plastic detectors of the
PM355 (PADC) type. The miniature scintillation detectors were placed in the detection plane of
the spectrometer, at different points upon the deuteron parabola, which corresponded to
determined energy values. The applied scintillation detectors were sensitive to fast ions and X-
rays pulses, but the recorded signals could easily be identified. The applied configuration of the
detectors allowed us to determine instants of the ion emission (using a TOF technique) and
compare them with instants of the X-ray emission.

First EPs Conference on Plasma Diagnostics - $1^{\text {st }}$ ECPD

14-17 April 2015,

Villa Mondragone, Frascati (Rome) Italy

$1 \quad$ Presenting author 


\section{Introduction}

Measurements of the ion beams emitted from a dense plasma region are of particular importance for the understanding of physical processes occurring inside the pinch column, but also for the determination of ion streams characteristics. Such ion beams can also be used for material engineering, e.g., for research on behaviour of different materials irradiated by intense plasma streams [1-3].

High-current pulse discharges within a PF-1000 facility have been studied for several years [2-5], but the previous measurements were focused mainly on shapes and structures of the investigated ion beams. In a frame of the reported study, to evaluate energies of the ions the use was made of a modified Thomson-parabola spectrometer. It was equipped with nuclear track detectors (used for time-integrated measurements) or miniature scintillation detectors (used for time-resolved measurements). The main aim of this study was to investigate dynamics of the ion emission from the investigated PF discharges.

\section{Experimental setup}

The reported measurements of fast ion beams were performed within the modified DPF1000U facility equipped with two coaxial electrodes of $460 \mathrm{~mm}$ in length. The inner electrode was a copper tube of $230 \mathrm{~mm}$ in diameter, while the outer electrode was composed of twelve 80mm-diam. stainless-steel tubes, distributed symmetrically around a cylindrical surface of 400 $\mathrm{mm}$ in diameter [5]. The inner electrode was equipped with a fast-acting gas valve which was located within the central hole. That valve was used to inject an additional amount of deuterium (about $1 \mathrm{~cm}^{3}$, at pressure of $1.5 \mathrm{bar}$ ) along the symmetry axis of the device, into the region close to the inner electrode end-plate. The investigated PF-type discharges were performed at the initial deuterium pressure equal to 1.6 or $2 \mathrm{hPa}$, and were supplied from a condenser bank charged to $23 \mathrm{kV}, 334 \mathrm{~kJ}$.

Characteristics of the ion emission were investigated by means of a Thomson-type spectrometer placed at z-axis inside the DPF-1000U vacuum vessel [6]. The input diaphragm of spectrometer was located at a distance of $125 \mathrm{~cm}$ from the electrodes ends (Figure 1).

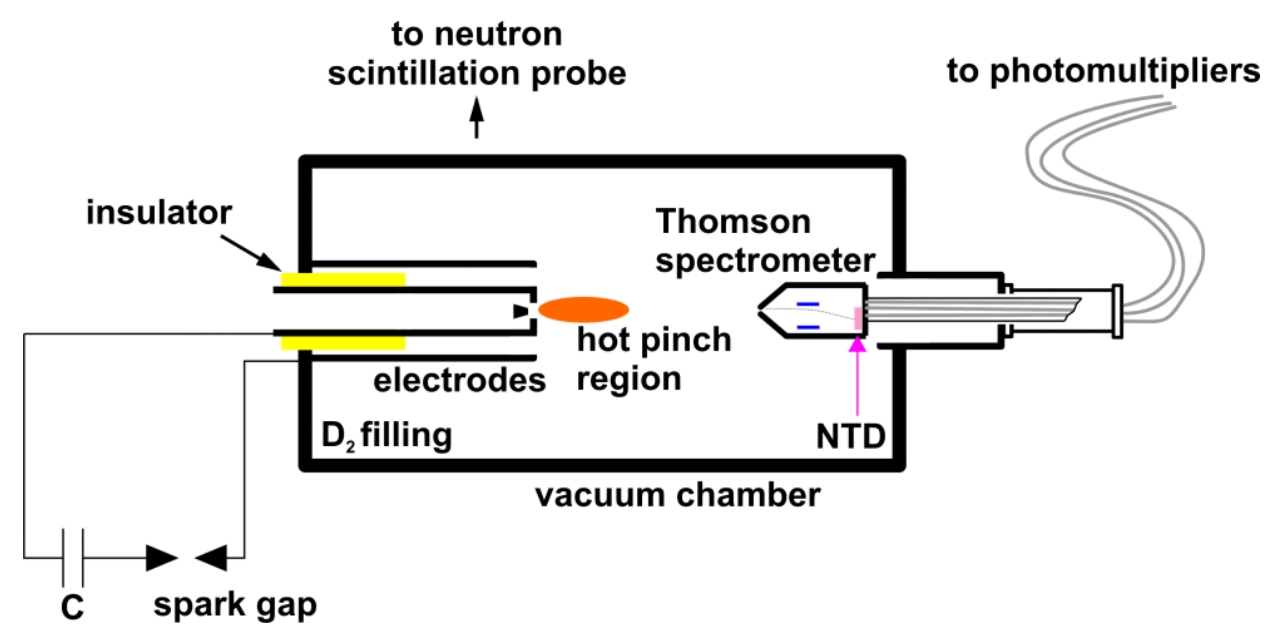

Fig. 1. Scheme of experimental setup during ion measurements carried out with the Thomson spectrometer located inside the DPF-1000U chamber. 
Energy spectra of the investigated ions were recorded by means of nuclear track detectors of the PM-355(PADC) type, which after irradiation and chemical etching at the standard conditions were investigated with an optical microscope [7]. The time-resolved measurements of the ions were performed by means of miniature scintillation detectors of the NE102 type, which were placed along the deuterium parabola, at positions corresponding to deuteron energies equal to $135 \pm 25 \mathrm{keV}, 100 \pm 20 \mathrm{keV}$ and $45 \pm 5 \mathrm{keV}$ (see Figure 2)

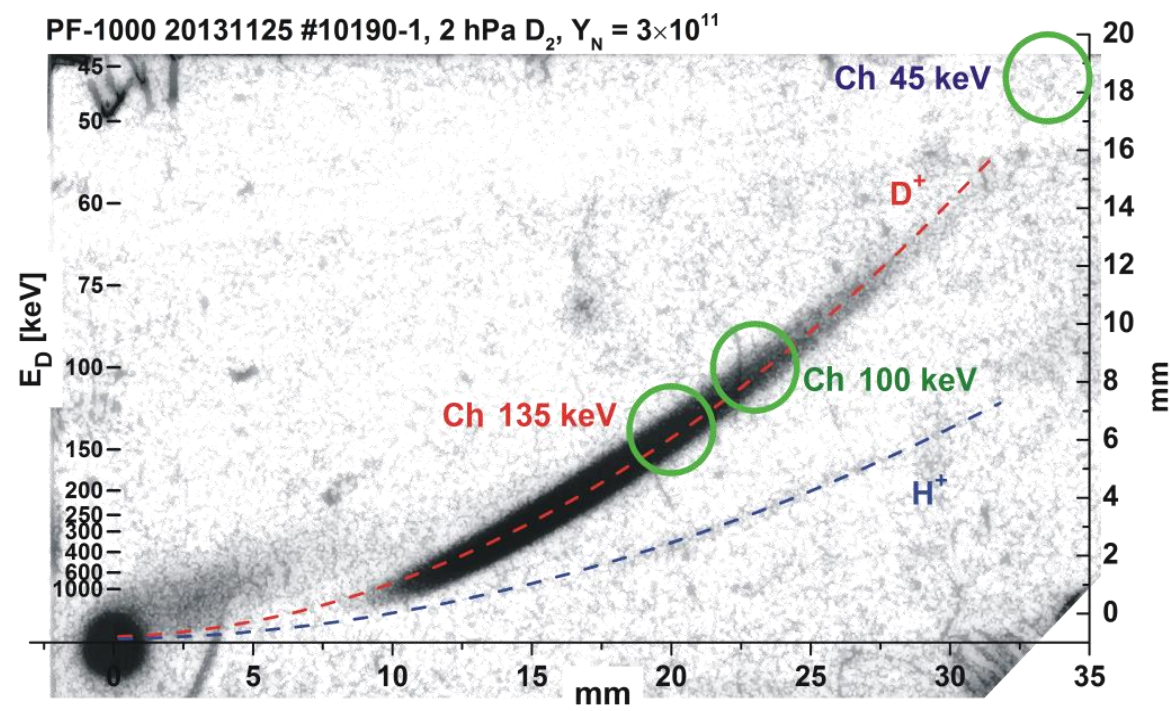

Fig. 2. Deuteron and proton parabolas recorded during two successive DPF-1000U discharges. Green circles mark the positions of scintillation detectors which recorded deuterons of the indicated energy values. The total neutron yield $\mathrm{Y}_{\mathrm{N}}$ was equal $3 \times 10^{11}$.

The applied scintillation detectors were coupled with fast photomultipliers through separate optic fibres, and signals were recorded with a digital oscilloscope. As a reference we used signals from a neutron scintillation probe, which was located outside the vacuum vessel. The period of discharge when the observed ions could be emitted from the pinch column, was calculated on the basis of a time-of-flight (ToF) method. Those calculations were performed under the assumptions that: a) ions could be emitted from different regions along the dense plasma column, b) the recorded ions had energies in a range determined by positions and sizes of the applied scintillators, c) the ions were slowed down by the gas filling [8].

\section{Experimental results}

Energy spectra of deuterons and protons emitted from a DPF-1000U plasma focus region were determined on the basis of recorded ion parabolas. The nuclear track detectors, which were placed inside the Thomson spectrometer, were irradiated during one or several discharges. The colour-enhanced deuteron parabola (plotted on the basis of microscope scanning of the detector surface) and energy spectrum, as calculated on the basis of the recorded ion image, are shown in Figure. 3. In that case the initial deuterium pressure was equal to $2 \mathrm{hPa}$, and the discharge was performed without using the gas-puffing. The accelerated deuterons emitted from the DPF1000U facility had energies up to $1.2 \mathrm{MeV}$. 
The time-resolved measurements of ions were performed by means of the same Thomson spectrometer, but equipped with miniature scintillation detectors coupled through separate optical cables with fast photomultipliers. Energy ranges of the recorded ions were determined by sizes of scintillators and spectrometer characteristics (its dimensions, as well as values of the applied electric and magnetic fields).
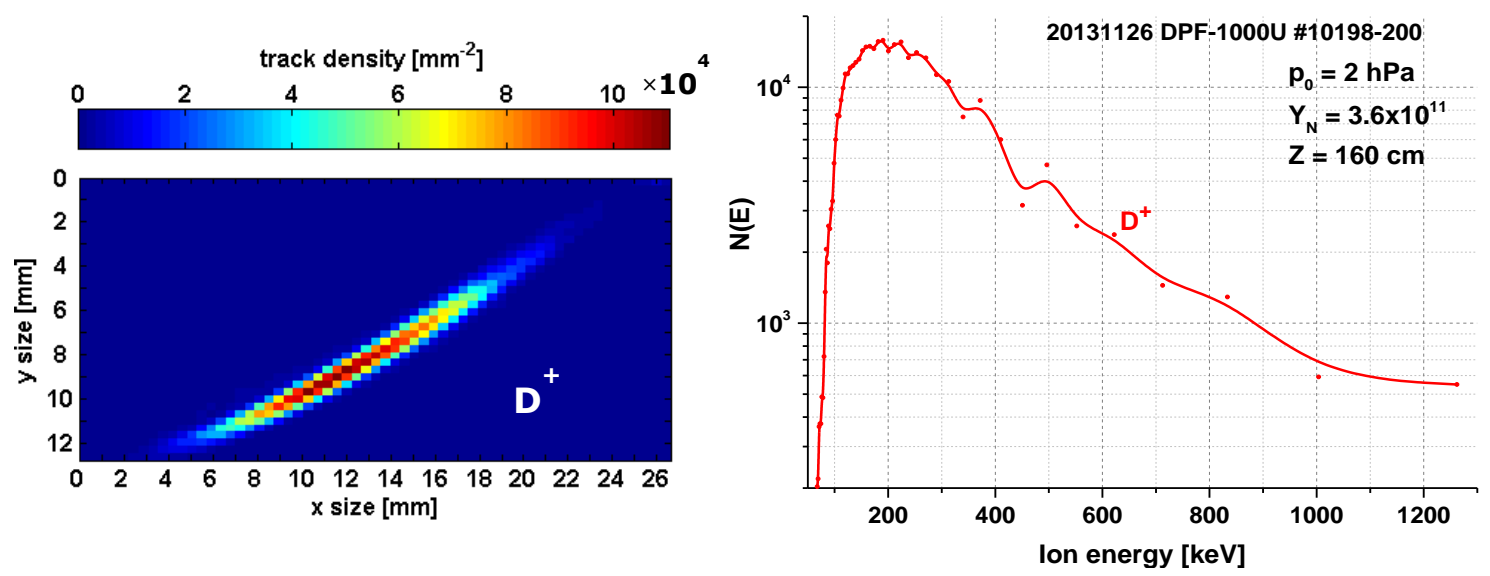

Fig. 3. Colour-enhanced image of the deuteron parabola (left) recorded during a discharge without any gas puffing and deuteron energy spectrum (right). The initial deuterium filling pressure was equal to $2 \mathrm{hPa}$.

Knowing the energy range of ions recorded in each measuring channel, it was possible to calculate time intervals corresponding to the generation of fast deuterons. The reported measurements were performed under various experimental conditions. Typical signals recorded during a discharge performed without any gas-puffing are presented in Figure 4. The colour solid lines (shown below the recorded traces) mark time intervals when ions (registered within a corresponding channel) could be generated.

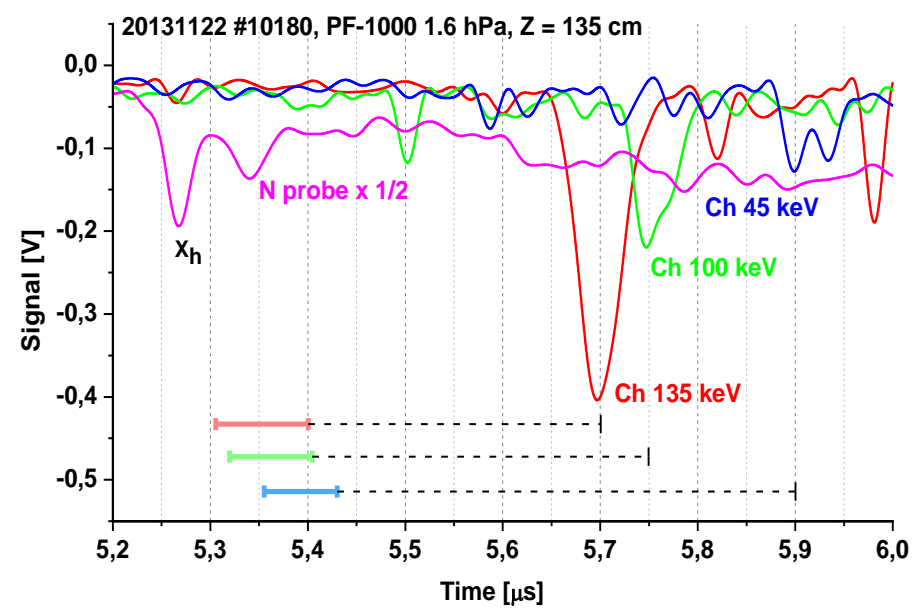

Fig. 4. Time-resolved signals recorded by means of Thomson spectrometer equipped with miniature scintillation detectors (different measuring channels $\mathrm{Ch}$ ). The initial pressure was equal to $1.6 \mathrm{hPa}$. The detector plane was placed at distance $135 \mathrm{~cm}$ from the ends of the electrodes. 
The ion peak recorded in "Ch $100 \mathrm{keV}$ " channel at the instant $\mathrm{t}=5.5 \mu$ s could be produced by fast ions which were slowed down by gas filling and reached energy about $100 \mathrm{keV}$ in a close proximity of the detector, or by ions generated several tens nanoseconds before the X-ray emission.

An example of the ion signals composed of multiple peaks is shown in Figure 5. In that case, there were marked time periods when ions (generated simultaneously with hard X-rays pulse) should be recorded.

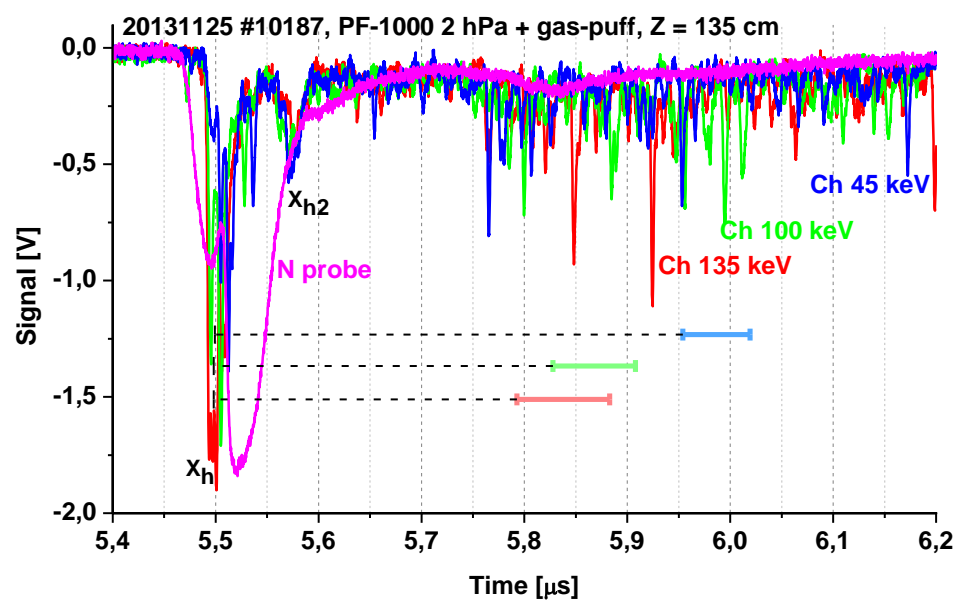

Fig 5. Signals recorded during a discharge with the gas puffing at initial pressure equal to $2 \mathrm{hPa}$. Detector plane was at distance $135 \mathrm{~cm}$ from the electrodes. The colour solid lines show time intervals when ions, generated during 1 st hard X-ray pulse, could be registered.

The signals of such a type could be produced by strongly scattered ions. In that case the ions could have various initial energies, but be recorded by the spectrometer as the ions of similar energy, at different instants. The multiple peaks could also be results of some perturbations in a diagnostic system, i.e., by light pulses generated inside optical cables or electric signals generated within the photomultipliers. The authors of this paper did not know a credible method to identify sources of these multiple peaks. Therefore, we have marked only probable periods when the considered ions could be registered.

\section{Summary and conclusions}

The described measurements of ions emitted from plasma focus discharges within the DPF-1000U facility, which were carried out by means of a Thomson parabola spectrometer, showed that energies of deuterons can amount up to $1.2 \mathrm{MeV}$. The analysis of the obtained timeresolved ion signals and time-of-flight calculations showed that deuterons of energies from about $50 \mathrm{keV}$ to about $150 \mathrm{keV}$ could be emitted up to about $150 \mathrm{~ns}$ after the hard X-ray emission. The used scintillation detectors were sensitive to X-rays, fast neutrons and ions, but the thicknesses of the applied detectors were below $1 \mathrm{~mm}$. Therefore, neutron-induced signals were weak in a comparison with the observed X-ray and ion peaks. The types of signals were identified by a comparison of the recorded signals from the Thomson spectrometer with those 
from a neutron scintillation probe, which was placed outside the experimental chamber. In particular, it concerned instants of the X-ray peaks appearance. It can be concluded that the recorded ion peaks originated from sources, which could be formed inside the pinch column almost during the whole period of its existence. It should also be noticed that in some discharges the emitted ions were strongly scattered and slowed down by a working gas inside the DPF$1000 \mathrm{U}$ experimental chamber, what resulted in the appearance of many ion peaks.

\section{References}

[1] L. Bertalot, H. Herold, U. Jager, et. al., Phys. Lett. Vol. 79A (1980) 389.

[2] E. Skladnik-Sadowska, J. Baranowski, M. Milanese, et al., Radiat. Meas. 34 (2001) 315.

[3] J. Zebrowski, M. J. Sadowski, K. Czaus, et al., Czech. J. Phys. 54 (2004) 643.

[4] L. Jakubowski, M. Sadowski, J. Żebrowski, Nucl. Fusion Vol. 41 (2001) 755.

[5] M. Scholz, L. Karpinski, V. I. Krauz, et al, Nukleonika Vol. 57, No 2 (2012) 183.

[6] M. J. Sadowski, K. Czaus, K. Malinowski, et al., Rev. Sci. Instrum. Vol. 80 (2009) 053504.

[7] M. J. Sadowski, A. Szydłowski, M. Scholz, et al., Radiat. Measur. Vol. 31 (1999) 185

[8] http://www.srim.org/ 